\title{
Improving root cause analysis through the integration of PLM systems with cross supply chain maintenance data
}

\author{
N. Madenas ${ }^{1 *}$, A. Tiwari*1, C.J. Turner ${ }^{1}$, S. Peachey ${ }^{2}$, S. Broome ${ }^{3}$ \\ 1 Manufacturing and Materials Department, Cranfield University, MK43 OAL \\ 2 Axillium Research, Unit 20, Cottesbrooke Park, Daventry, NN11 8YL, \\ 3 Jaguar Land Rover, Abbey Road, Whitley, Coventry CV3 4LF,
}

*Corresponding author: N. Madenas, Manufacturing and Materials Department, Cranfield University, MK43 OAL, Nikolaos.madenas@cranfield.ac.uk; Tel: T: +44 (0) 1234758250

\section{A B S T R A C T}

The purpose of this paper is to demonstrate a system architecture for integrating Product Lifecycle Management (PLM) systems with cross supply chain maintenance information to support root-cause analysis. By integrating product-data from PLM systems with warranty claims, vehicle diagnostics and technical publications, engineers were able to improve the root-cause analysis and close the information gaps. Data collection was achieved via in-depth semi-structured interviews and workshops with experts from the automotive sector. Unified Modelling Language (UML) diagrams were used to design the system architecture proposed. A user scenario is also presented to demonstrate the functionality of the system.

Keywords: Root cause analysis; Warranty; Supplier Integration; Product Lifecycle Management; Information systems;

\section{Introduction}

The International Journal of

Advanced Manufacturing

In large and complex automotive supply chechnology, May 2016, Volume 84,

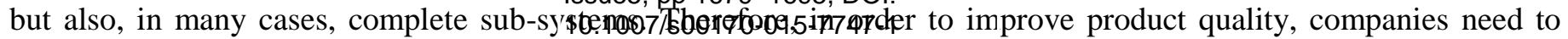
extend their area of analysis and decision making to include not only their internal business units but also their supply chain. Recent advances in IT have enabled companies to improve supply chain collaboration and share more accurate information. However, the vast amount of systems used to design, develop, produce, deliver and support products for global markets have created a major barrier in information sharing. Recent investments on product lifecycle management (PLM) systems demonstrate how important it is for manufacturers to improve supply chain collaboration as well as store data in a central location. Although PLM systems offer a centralised location for maintaining and managing product data, their focus is still fragmented within the product development phase of the product life cycle. The integration of maintenance information such as vehicle diagnestics, warranty claims and technical publications with PLM systems will allow information and knowledge to be reused both by product development (PD) engineers and by warranty engineers. As information sharing is the enabler for improving the decision-making process and the entire supply chain performance, it is important not only to determine the level of access for suppliers but also to determine how processes are integrated and what information should be made available to suppliers.

Root cause analysis is one of the areas in the automotive sector where data integration and automation are absolutely critical. Root cause analysis within the automotive sector has become more complex due to the number of participants involved, the volume and the structure of information required, and the high number of interrelated functions that are impacted both upstream and downstream in the value chain. During the warranty period, identifying the root cause of a particular in-service product failure and developing knowledge such as trends and patterns between product failures and warranty claim attributes is key in achieving warranty cost reduction [1]. In the large and complex automotive value chains, it is estimated that warranty costs are around $3 \%$ of the original equipment manufacturer (OEM) revenue [2]. Although vehicle manufacturers were traditionally focusing on introducing new vehicles in the market as early as possible, they are now concerned about the increased warranty costs which can have an impact on both customers' satisfaction and brand reputation. Mannar et al. [3] state that although studies in the area of warranty analysis extract important information related to lifetime and failure rates, they do not demonstrate how these data could feed back to manufacturing or design in order to identify interactions between design changes and warranty failures. The use of maintenance information such as vehicle diagnostics from the sensors, warranty claims and technical publications in relation to design data from the product life cycle management (PLM) systems could potentially offer significant benefits.

The purpose of this paper is to demonstrate a system architecture for integrating product lifecycle management (PLM) systems with maintenance information to support root cause analysis by allowing engineers to visualise cross supply chain maintenance data in a single environment. Section 1 introduces the research area while Sect. 2 presents 
the previous research. Section 3 presents the research methodology followed. Sections 4 and 5 present the outcome of this research by demonstrating the current state of root cause analysis and proposing a novel system architecture to address the existing challenges. Sections 6 and 7 present and summarise the key findings of this research.

\section{Relevant Research}

During the last decade, an extensive body of literature has been formed to improve maintenance and support efficiency. The use of technology and especially web-based applications has allowed the movement from traditional models of maintenance to e-maintenance [4]. To address the upcoming challenges, researchers are focusing on developing methodologies and tools to enable full utilisation of the product data and information in processes [5]. Although web-based technologies have significantly improved communication among supply chain members, there are still many challenges to be addressed. One of the main challenges that companies are currently facing is the lack of a central repository to manage and maintain maintenance data. Until recently, organisations have developed multiple internal systems to manage different types of information and, as a result, they now face the need to develop standards and integrate those systems.

An area that has received significant attention during the last decade is that of PLM systems mainly due to the potential they offer for improving supply chain collaboration. Although PLM systems are defined as a strategic business approach that supports collaboration from concept to end of life [6], PLM systems still remain very isolated from the maintenance procedures. Lee et al. [7] state that PLM systems are used 10 times less frequently in the maintenance phase and highlight that the potential of PLM systems in Maintenance Repair Overhaul (MRO) has not been realised. Gulledge et al. [8] proposes a solution for integrating PLM systems and condition-based maintenance in order to convert prognostic and diagnostic information into actionable information that can be directed into PLM environment using service oriented approaches. Candell et al. [5] emphasise the fact that ICTcan be the enabler to improve effectiveness and propose a service-oriented eMaintenance platform to support the complex maintenance processes. Sairamesh et al. [2] proposes a solution to allow OEMs and dealers to work collaboratively through contextual collaboration and information integration using service-oriented approaches. The solution proposed aims to integrate 3D parts catalogues with warranty processes and purchasing processes.

\subsubsection{Warranty Analysis}

Regarding the data analysis aspect, the warranty area has been researched widely. Djamaludin et al. [9] listed more than 1500 articles in this area. Recent reviews can be identified in the literature. $\mathrm{Wu}$ [10] categorises the different types of "coarse" warranty data and reviews relevant techniques in analysing those data. Karim and Suzuki [11], through a literature review, classify the analysis of warranty data in nine categories based on the different statistical models and methods used. The study of $\mathrm{Wu}$ [12] is an excellent review article that clusters the different warranty analysis techniques in five areas.

Over the last 40 years, several studies have been published and propose statistical models and methods to analyse warranty claims based on different attributes and factors such as manufacturing conditions or the environment where the product is used [13], hazard rate from incomplete warranty data [14] or even warranty policies for non-repairable items [15]. The outcome of these studies focuses in improving product reliability, supporting product design decisions and managing warranty policies. Similarly, several publications proposed models in order to forecast the expected cost of warranty claims [16-19]. Another area that has received significant attention, especially during the last decade, is that of data mining techniques mainly due to the complexity and the amount of data involved in the process. Researchers propose algorithms to support OEMs in structuring warranty claims which in many cases are returned as unstructured data $[1,20,21]$. Choudhary et al.'s [22] literature review discusses the different knowledge discovery and data mining techniques used in the area of manufacturing. Sureka et al. [21] present a rule-based text analysis prototype system which was developed to support automotive warranty analysts in obtaining better understanding into product defects reported from the dealer. As Buddhakulsomsiri et al. [1] state, "unless the IT systems are designed to capture the desired information and can easily piece together the disparate pieces of data, the quality of the knowledge extracted from the data may be uncertain". Buddhakulsomsiri et al. [1] propose a new rule generation algorithm for mining automotive warranty data that aims to identify relationships between product attributes (production rate, repair date, mileage at repair, etc.) and the decision outcome.

\subsubsection{On-board Fault Diagnostics and Prognostics}

During the last decade, technologies related to fault diagnostics and prognostics systems especially in the aerospace and the automotive sectors have become available. The on-board fault diagnostics and prognostics systems are responsible for continuously monitoring the critical operation of each system. In case of a malfunction, the system 
generates a Diagnostic Trouble Code (DTC) which is then stored within the on-board computer of a vehicle [23]. DTCs generated over a period of time can be extracted by dealers using the appropriate software tool. This information provides insights into the nature of the fault as well as assistance in the determination of corrective actions that need to be performed on the vehicle [24]. One of the most important benefits of the on-board fault diagnostics system is that it captures data that are not subjective to the technician's or the customer's interpretation, but represents data captured in real time from the sensors. Gusikhin and Rychtyckyj [25] provide a review study on the different ways that the automotive industry has utilised artificial intelligent (AI), soft computing and other intelligent system technologies in multiple areas including warranty analysis and design. Similarly, Venkatasubramanian [26] provides an overview of the various approaches to automated fault diagnosis and highlights current challenges and future opportunities. Several studies have been identified in the literature that utilise data captured from vehicle sensors to support decision-making. Mannar et al. [3] propose a methodology for identifying relations between warranty failures and manufacturing measurements in order to diagnose warranty failures and perform tolerance revaluation. This study provides an extensive discussion in the area of field performance information. Khare et al. [27] using real-time diagnostic data collected from Telematics demonstrated that (i) the accumulation of miles is approximately linear with age and (ii) the distribution of mileage accumulation rate in vehicles with no claims is the same as that with at least one claim. Liu et al. [28] developed a decision model to support product design management to estimate the cost of designing diagnostics against the benefits and the warranty cost reductions that the designed diagnostics will bring. Chougule et al. [24] developed a novel approach for integrating warranty claims and vehicle diagnostics to support engineers in identifying anomalies in the field, performing root cause analysis and capturing training needs for dealers. Rajpathak [23] proposes a novel ontology-based text mining system in order to analyse unstructured textual diagnosis data collected from automotive OEMs during the warranty period. Saxena et al. [29] propose a hybrid reasoning architecture for integrated fault diagnosis and health maintenance of fleet vehicles to support maintenance decisionmaking and capture knowledge.

\subsubsection{Research gap}

Literature review has shown that PLM systems still remain very isolated from the maintenance procedures. Although a few researchers proposed ways of integrating PLM systems with maintenance information, most of these studies are still related to the aerospace sector and less work has been done within the automotive sector. The outcome of the literature enhances Lee et al.'s [7] results which showed that PLM systems are used 10 times less frequently in the maintenance phase and their potential in MRO has not been realised yet. In addition, literature review in the area of warranty analysis showed that all the models proposed and the data mining techniques discussed either focus on a single data set or assume that the data sets analysed are stored in one database and therefore the algorithm can be easily applied. Buddhakulsomsiri et al. [1] state that "unless the information technology systems are designed to capture the desired information and can easily piece together the disparate pieces of data, the quality of the knowledge extracted from the data may be uncertain". The integration of PLM systems with maintenance information such as warranty data and on-board vehicle diagnostics will allow the utilisation of knowledge and data cross the product life cycle and support not only internal decision makers but also the external supply chain. In addition, it will allow aftersales information and knowledge from maintenance and service experts to feed back to the product development process

\section{Methodology}

Data collection was achieved through a combination of semi-structured interviews with experts from the automotive sector, by studying the internal documentation and by testing the systems used. Using a combination of data collection techniques provided validity and reliability to the results and reduced any potential bias. All interviews arranged were face to face and included people from various roles such as quality managers, warranty managers and service engineers for the warranty use case, and PD engineers and managers for the Design Failure Mode and Effect Analysis (DFMEA) use case. In addition, quality data managers and IT system architects were also interviewed for understanding the infrastructure and the systems involved in the process. Interviewees included engineers from a major vehicle manufacturer and four tier 1 automotive suppliers. All companies were global and have a UK presence. Semi-structured interviews were deemed as the most appropriate technique for collecting data due to the type of data required for this research.

Data collection involved 27 semi-structured interviews for capturing the current state and as an extent the business processes, the information systems and the information used. In addition, 21 semi-structured interviews were conducted to capture the future state, analyse and prioritise the requirements, and design the system architecture. In 
several cases, interviews were conducted with a group of people in order to include technical IT people and engineers who are the end users. The questions for each semi structured interview were adjusted based on the role of each interviewee. For example, the warranty spend reduction leader was asked questions related to the process, the decision points and the systems used while the quality data manager was asked questions related to the integration of the systems, the database structure, inputs and outputs. The outcome of each interview was formally documented and sent back to the interviewees to review and comment. This approach ensured that any potential bias was minimised as each interviewee reviewed and validated the outcome (Fig. 1).

Once the requirements were analysed and prioritised, Unified Model Language (UML) diagrams such as use case diagram, sequence diagrams and package diagrams were created to transform user requirements into system functionality. As part of the requirement elicitation process, experts supported the prioritisation of requirements using the MoSCoW approach. By engaging key stakeholders within the prioritisation of requirements ensured that any potential bias was minimised. UML diagrams were also used as a communication method between stakeholders and system developers and as a validation point throughout the development of the system. The methodology used ensures that the system developed satisfies business requirements and as a result brings benefits to the business and its supply chain.

The system architecture was tested and validated by OEM and supply chain engineers. Semi-structured interviews were deemed as the most appropriate technique of validating the outcome of the proposed system architecture. All semi structured interviews arranged were face-to-face and lasted on average $90 \mathrm{~min}$. High-level scenarios in conjunction with screenshots and a small video from the system were used to demonstrate (i) how the user interacts with the system, (ii) how the system aggregates the data, and (ii) what the system presents to the end user.

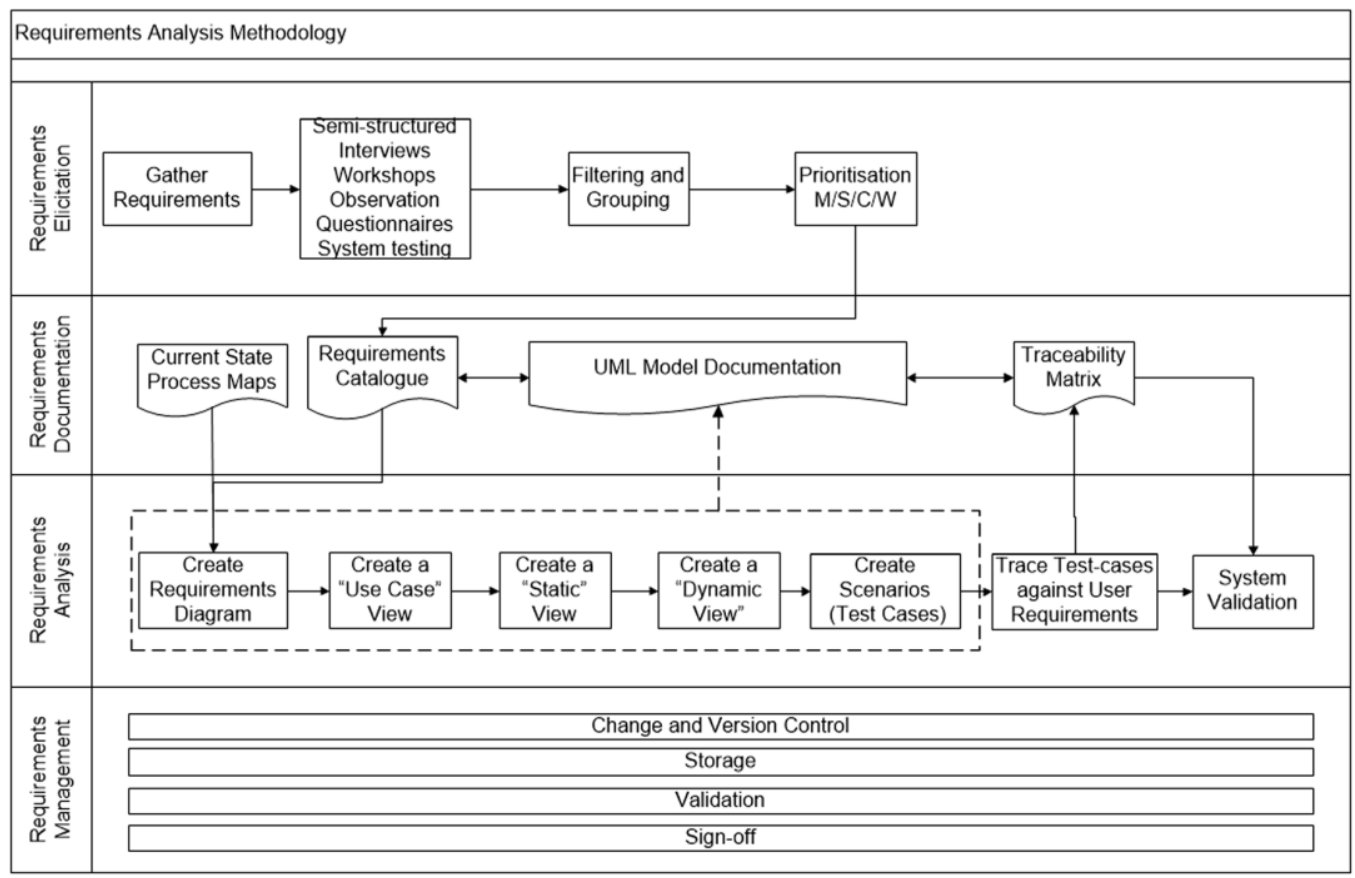

Figure 1 Research Methodology

\section{Results - Current State process}

The first part of the methodology analyses two processes where automotive vehicle manufacturers and component suppliers utilise maintenance data to conduct root cause analysis, using data collected from 27 semi-structured interviews with experts from the automotive sector. The following two processes are demonstrating the areas that the outcome of the proposed system will support. The first process demonstrates the flow and the use of in-service product failure data, such as warranty claims and vehicle diagnostics, reported from a service representative (dealer) back to the OEM and then to the component supplier to conduct a root cause analysis of a product failure that occurred on a specific vehicle. The second process shows the utilisation of maintenance data to produce a comprehensive list of past failures, concerns and their root causes to support PD engineers to understand previous faults prior to the design of a new part or sub-system. Challenges identified in each part of the process will scope the requirements analysis and base the development of the system architecture. The current state process maps capture more information than the proposed solution could achieve but demonstrate clearly areas where the system architecture will support. 


\subsection{Root Cause Analysis process - In-service failure}

\section{Step 1: Fault identification and repair}

The root cause analysis process is triggered when a customer identifies an issue and reports to the dealer to resolve. Capturing what the customer complaint is about is key as the customer's feedback feeds into the OEM's warranty system and provides an initial understanding of the fault. Once the dealer captures the customer's feedback, the next step is to identify the part failure. Using a variety of information from multiple systems such service manuals, technical service bulletins, instructions provided from the vehicle manufacturer and diagnostics extracted from the vehicle, the dealer will determine the failure part and make a repair. Following up on the repair procedure, dealers are responsible for recording a claim in the OEM's warranty system. Once the claim is recorded in the warranty system, dealers either scrap the part or return the replaced parts to the OEM's testing centre for further analysis.

\section{Step 2: Supplier Root cause analysis}

The analysis of the returned parts provides great insights for understanding systematic failures and identifying the root cause of an issue. It allows vehicle manufacturers to have a close view of the part and if required to recreate the failure conditions in a testing vehicle. It also allows suppliers to compare the results of a testing conducted to the returned part with the quality data obtained before the part was initially shipped to the OEM. As a result, they can see degradation trends among different markets and conditions. All the data captured from each system are used by the component supplier to assess a part and identify the root cause of an issue. Some of the questions that suppliers need to answer are as follows: (i) Was this a known issue? (ii) Was it the causal part? (iii) Did the dealer have enough information to make a repair? (iv) Were the instructions followed? The following information is considered key for any warranty root cause analysis:

- Vehicle's history is important as previous in-service failures might have an impact on the latest claim. It will also show the supplier the conditions and the markets that the vehicle was operating in.

- Breakdown recovery reports give a detailed view of the conditions of the fault.

- Vehicle diagnostics captured through the vehicle control unit are used to understand the conditions that the vehicle was operating in and what the sensors recorded during the occurrence of a fault (mileage, temperature, DTCs, etc.)

- Supplier test data captured from tests conducted to the returned part from suppliers are also important to understand how the part is currently operating compared to how the part should operate.

- Product-related data held in PDM/PLM systems such as CAD models, BOM, specifications and documentation associated with the part.

\section{Step 3: OEM assessment and validation}

Further to the investigation conducted by suppliers, OEM engineers are investigating no trouble found (NTF) issues further in order to ensure that suppliers' testing methods meet the expected test criteria. What the engineers are looking for is to ensure that the testing criteria and the data used are sufficient to replicate the symptom and identify the root cause of the issue. The following questions are examples of what the OEM engineers are looking to assess: (i) Is symptom and failure mode understood? (ii) Does supplier assessment check for each failure mode? (iii) Does assessment replicate actual installation environment? (iv) Are specifications compatible with customer usage cycle? (v) Did the dealer have enough information to make a repair? OEM engineers will analyse the returned parts and the claims and raise projects to the most relevant department such as engineering, manufacturing, service or even a supplier.

\subsection{Quality History for re-designing new parts}

Quality history offers an important input in the DFMEA as it utilises various information sources to produce a comprehensive list of past failures, concerns and their root causes based on internal and external data. Quality history is capturing not only hard failures which relate to parts that did not operate within the desirable objectives but also soft failures which relate to parts that operated within the desirable objectives but still failed to meet customers' expectations. Engineers consider the following as failures: (i) It does not do what it should do, (ii) it does something it should not do, and (iii) it does less than the customer was expecting. This process is triggered when the PD engineer is required to conduct a DFMEA analysis prior to the designing of a new part. The scope of the DFMEA can be either on 
a system, on a subsystem, or on a component level:

- Collate generic programme history: The purpose of this set of tasks is to obtain generic programme history from previous programmes that are relevant to the DFMEA scope. This task allows engineers to collate programme information such as stop shipments and vehicle recalls in order to understand critical system failures.

- Collate claims history for system, subsystem, or component: The level of granularity is defined from the DFMEA scope and aims to reveal trends and patterns in hard failures. In order to reduce the amount of data that needs to be analysed, warranty data can be filtered based on the vehicle's period in service. Pareto charts are used to group the different failure codes against the associated cost in order to have a summary view ofthe claims history.

- Collate voice of the customer data: The "voice of the customer" data refer to the surveys that customers provided and are used to reveal trends and patterns in soft product failures. PD engineers want to identify parts and subsystems that operated within the desirable objectives but still failed to meet customers' expectations. Usually, OEMs acquire surveys from third-party companies that focus on key markets across different vehicle periods.

- Collate vehicle diagnostics data: This data source was highlighted as key in understanding hard failure trends and patterns, especially for electronic failures. Similarly with the warranty claim analysis, DTC captured from the sensors can be used to identify or predict system failures. Hierarchical charts can be used to group DTCs for a selected period against the number of appearances in order to create a summary of diagnostic signals.

\subsection{Challenges}

The way the issue was communicated, analysed and captured can vary significantly among multiple dealers, especially from dealers recoding issues in their native languages. Key words and phrases used when recoding the customer's verbatim can offer great insights for the OEMs and component suppliers when analysing the root cause of a claim. In several cases, dealers failed to identify the most up-to-date technical service bulletin within the system, and without following the proper instructions, they were unable to resolve entirely the fault, which can lead to either an increased cost due to the replacement of the wrong part or customer dissatisfaction in cases where customers had to revisit the dealer in a short period of time. Identifying gaps in bulletins and diagnostics is an area that interviewees highlighted as a challenge which needs to be resolved as early as possible for reducing the warranty cost and improving customer satisfaction.

Accessing all the systems involved is currently a great challenge as identifying the information required is a very manual and time-consuming process. The depth of the information used in each review usually depends on the skills and the experiences of the engineer involved. Accessing data held within the PLM system is currently limited as warranty and quality engineers usually have only restricted access to the system. Usually accessing the multiple systems used is very manual and in many cases very resource intensive. Similarly, accessing data held within the PLM systems is currently a very manual process as warranty and quality engineers are usually restricted from accessing the PLM system. Again, the amount of effort required in terms of people and time is significant. Similarly to supplier engineers, OEM engineers stated during the interviews that identifying gaps in bulletin and diagnostics that dealers use is key in reducing NTF faults.

PD engineers highlighted that the number of systems and their complexity create obstacles in the process and causes issues when gathering the amount of details required to run a robust DFMEA. Similarly, with the in-service process, the quality history process is very manual and time-consuming and in many cases is subjective to the engineer's level of skills. The lack of in-depth quality history can cause misinterpretations when prioritising failure modes and effects. In many cases, engineers highlighted that without a robust quality history, the potential causes written within the DFMEA can be vague and subjective which can have a significant effect when measuring potential causes against severity and occurrence. Interviewees mentioned that the approach followed for each business unit and each supplier is different as there is no standardised process for capturing quality history. Moreover, in several cases, engineers were unable to find the appropriate amount of information required for the DFMEA, although they have accessed all the systems that the process defines. Interviewees highlighted that there needs to be a way to allow PD engineers to review those data without the need to fully access the system.

\section{$5 \quad$ Results - System Architecture}

The second part of the methodology presents the modelling techniques used to transform user requirements into system functionality. This phase demonstrates, through the use of three different architectural views (use case, static, dynamic), the interactions among the end users and the proposed system, the structure and the dependencies between 
the different elements of the architecture, and the sequences of tasks that occur within the system over time. The outcome of this phase defines the functionality required and demonstrates how the proposed system architecture will address some of the challenges identified within the processes discussed in Sect. 4.

\subsection{Use-case view}

The use case diagram describes the set of actions that the proposed system should perform in collaboration with one or more external human or system actors. Figure 2 shows the generic use cases that need to be completed by every user, the use cases related to the administrator of the system and the business cases that are relevant to the engineers that represent the end users of the proposed solution. Sequence diagrams are also provided to model the sequence of tasks for the two discussed processes. Basic flows are provided for the three use cases. The "Login", the "Register silos" and the "Define workflows" use cases follow the standard IT flow that is used for any system designed and therefore are not explained in detail.

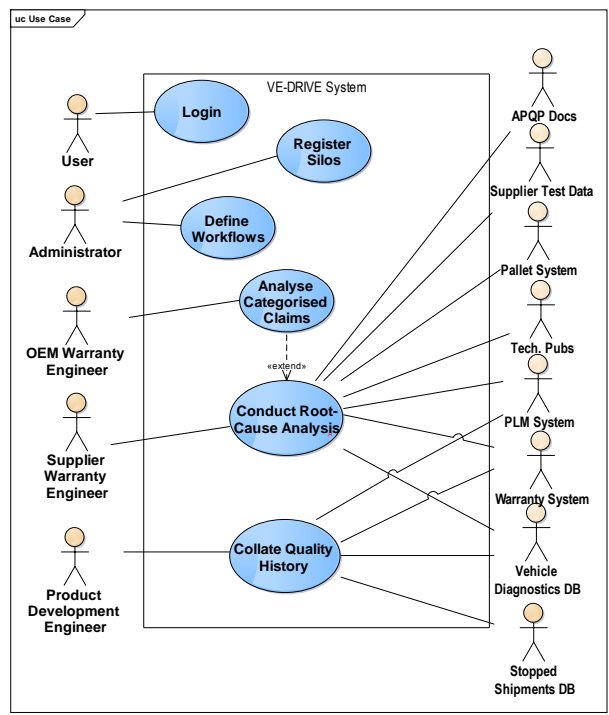

Figure 2 Use Case Diagram

\subsubsection{UML Use Case - Conduct Root Cause Analysis}

This use case is initiated when a component supplier is ready to assess a number of returned parts held in a pallet as described in step2 in the current state section. Supplier engineers shall be able to conduct a root cause analysis, assess a returned part and assign a liability (supplier fault, OEM fault, NTF, incorrect repair). To perform this task, engineers should gather, at minimum, the data described in the current state process. The outcome of this use case will allow warranty engineers to identify where gaps in the dealer diagnostic, repair or root cause analysis procedures exist and as a result close the information gaps and reduce the time to detect and resolve an issue. The use case is successfully completed when the user has reviewed all the information and assigned a liability against a claim.

Basic flow: Figure 3 expands on the use case described by showing all the steps followed in order to satisfy the use case. Supplier warranty engineer defines the search criteria using a VIN number. The system then requests and presents the vehicle description, "voice of the customer" data and vehicle's claims from the warranty system. In addition, the system presents a visual representation of the vehicle highlighting areas where claims occurred. Once the supplier warranty engineer understands the conditions that the car was operating and the history of the car, he/she selects the claim related to the reviewed part. The system extracts a CAD representation of the sub-assembly using the part number and the sub-assembly code from PLM system. In addition, the system extracts the PSW status using the part number from APQP Docs, any test data from the linked supplier's systems and DTCs from the vehicle diagnostics database using the VIN number and the time period. Finally, the system opens the Tech. Pubs application to the appropriate page using the VIN number. The system presents all the information and allows the user to interact with the data by selecting the appropriate menu option. In the end of the session, the supplier engineer records actions and notes of the session. These steps can be repeated multiple times until the user has completed the pallet review. 


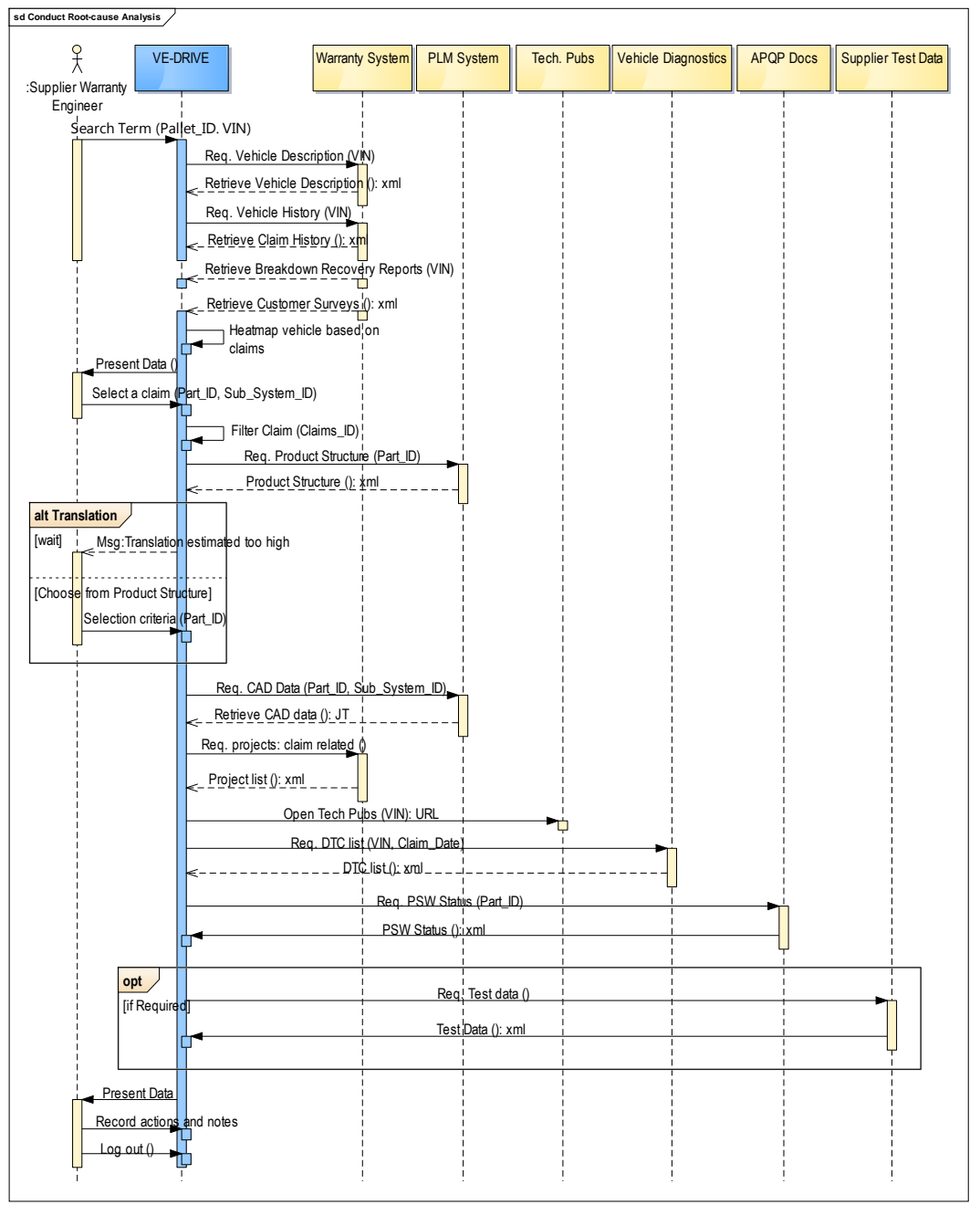

Figure 3 UC Realisation - "Collate Quality History to Identify the Root Cause of a Claim"

\subsubsection{Analyse Categorised Claims}

The system allows the OEM warranty engineers to assess the categorisation of claims by suppliers as described in step 3 in the current state section. Primarily, OEM warranty engineers are looking for suppliers that have a high number of NTF claims. Another criterion that OEM engineers track is the amount of warranty spend against the failure occurrence. This use case is initiated when OEM warranty engineers identify repetitive NTF claims associated with a similar failure effect. Using the same data that suppliers reviewed, OEM engineers need to validate that suppliers were able to replicate the conditions when the part failed; the test methods that suppliers used meet the expected levels and finally ensure that the dealers had enough information to make a repair. This use case is successfully completed when the user has reviewed and analysed all the required information. The outcome of this session will be either an acceptance of the supplier's decision or a start of a project for further investigating the issues identified.

Basic flow: The sequence of tasks followed in this use case is the same with the one described in the "conduct root cause analysis" use case. Although the steps in terms of system flow are the same, the decision points and the way that data are analysed are different as explained within the process.

\subsubsection{Collate Quality History}

The PD engineers shall be able to utilise various information sources to produce a comprehensive list of past concerns or failures and their root causes, based on internal and external data. The purpose is to allow engineers to run a robust DFMEA process and as a result prevent recurring of previous faults. This use case is initiated once the scope of the DFMEA is defined. The user needs to obtain the quality history required for the DFMEA prior of designing a part or a subsystem for a new vehicle. This use case is successfully completed once the user has reviewed and analysed all the information required to determine and assess each failure modes. 
Basic flow: The PD engineer defines search criteria using either the Function Group code, the Sub-assembly_code or the Part_ID. The system requests the product structure from the PLM System and warranty claims, breakdown recovery reports and surveys from the warranty system. To reduce the amount of data returned, the system needs to extract data for both 3 months-in-service (MIS) and 3 years-in-service (YIS) vehicles. In addition, the system extracts DTC from vehicle feedback database using the module_ID and the dates defined, and stopped shipments data associated with the searched programme. The system presents the returned results and the name of the systems where the data were extracted from. The PD engineer filters the data based on markets, months in-service or programmes. The system allows the user to create Pareto charts or hierarchical charts for each one of the data set extracted. The user concludes the use case by recording actions and notes of the session (Fig. 4).

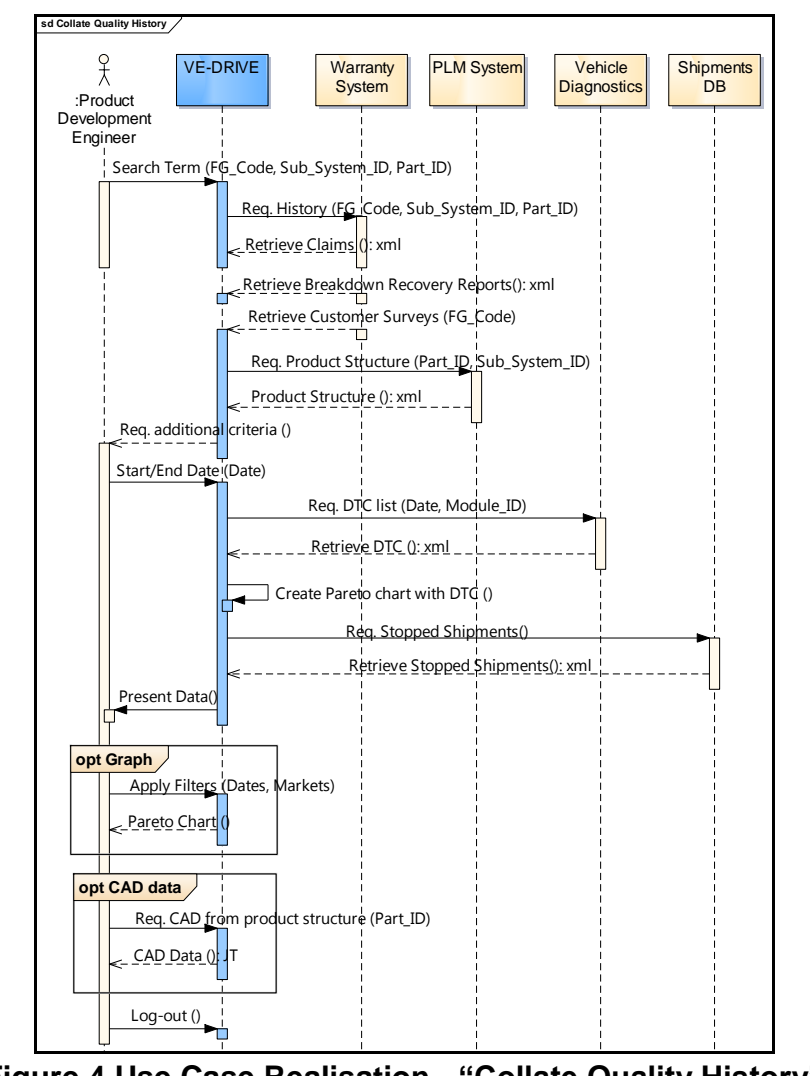

Figure 4 Use Case Realisation - "Collate Quality History"

\subsection{Static view}

Static analysis demonstrates the structure of the proposed system by decomposing the high-level architecture into layers and packages. The static view creates a baseline in which the dynamic view will expand to include the dynamic behaviour of the system over time. Package diagrams and class diagrams are widely used to demonstrate the static view of a system architecture.

Figure 5 shows the high-level architecture divided into three layers: presentation, integration and domain. The core of the solution architecture is based on the premise that discrete silos of information can be made available via web services. Central to the system architecture is an aggregator that assembles the selected data and initiates translation services whenever required to improve the performance and meet the needs of the presentation layer. System adapters were developed to communicate the integration layer with data extracted from the domain layer. Web services were selected as a method of communication between the integration and the domain layer, due to their ability to support interoperable machine-to machine interaction and to manage the transfer of data securely. Data retrieved can be presented in multiple visual environments such as immersive environments, desktops and tablets.

Each one of the layers presented in these figures is autonomous and can be implemented as a physically separated component. This aspect is very important as it allows the reusability and agility of the system. Each layer can be developed separately and then linked with the layer below. Packages presented in Fig. 5 were developed as generic as possible to support more use cases from the automotive sector as well as use cases from other sectors. However, elements developed to satisfy specific requirements and as a result support specific processes are not meant to be reusable. Key aspect of the layer segmentation is to separate the domain layer which represents the business logic with the other layers that represent the technology required to extract and visualise the data. As a result, depending on the circumstances, this approach allows either the technology to be improved over time or the domain layer to be 


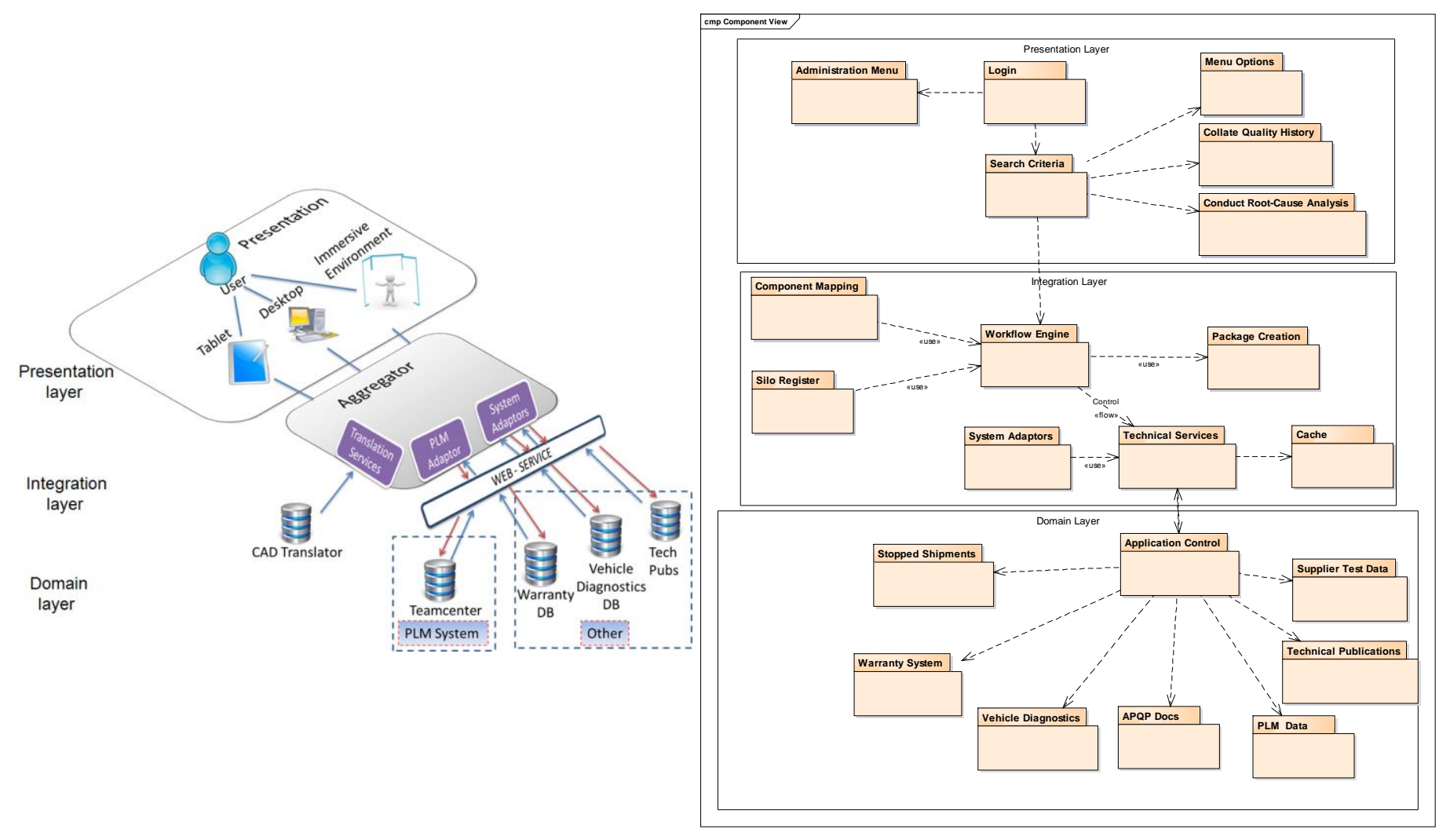

Figure 5a High-Level architecture

Figure 4b System architecture component view

\subsubsection{Presentation Layer}

The presentation layer consists of all the packages that construct the user interface (UI) and all the controls for user input and display selection. Within the presentation layer, there are seven packages that define the UI. The "menu options" package includes the different sets of UI controls. The login package represents the login interface in which users will provide their username and a password. The search criteria component represents the search input to the system which is the trigger of the workflow engine and the link to the integration layer. The search could be either a VIN number, a part number or a subassembly code. The input to the search element could be either through the use of a keyboard or through QR code scanned from a portable device. The user interface is specific to the end users and can be modified depending on the use cases and the specific requirements.

\subsubsection{Integration Layer}

The integration layer can be characterised as the "glue" between the domain and the presentation layer. The integration layer is used to enable and manage the communication among different systems. The purpose of the integration layer is to automate the business processes involved and provide a unified access to information spread across discrete silos of information. The integration layer utilises web services to communicate and extract data from the domain layer. A service-oriented integration layer allows companies to access the system through heterogeneous IT infrastructures. The application exposes some of its functionality as a service without revealing any implementation details to the outside world [30]. Service interfaces are developed using XML Web services. The key components are explained below. The dynamic view presented in Sect. 5.3 explains how these components are linked and interact within the system.

Component mapping which can either be provided as a service or linked as a database specifies the relationships among different primary keys and provides an input to the workflow engine. The purpose of this element is to provide a clear definition of each primary key used within the systems integrated and to specify the relationships among the different data sets used. It provides an input to the workflow engine as it holds all the key information for integrating all the relevant data.

Workflow engine: The workflow engine holds the logic behind the system as it is responsible for defining the sequence of tasks required to perform a use case. The workflow engine is responsible for executing the sequence of tasks required to satisfy each one of the three main use cases defined. The workflow engine uses inputs from the component mapping and the silo register which holds all the information related to the systems integrated. The 
outcome of the workflow engine is an evidence pack that holds all the information requested through the UI and the search criteria defined.

Technical services: Technical services are responsible for a variety of tasks. Most importantly, they are responsible for analysing, decomposing and executing the queries in order to retrieve data from the domain layer. Whenever a user hits the search button, the system makes a call through the technical services to extract the appropriate files. The aggregator processes the results in order to save the file and then write information to the screen. The function returned from the web service is an XML file (created from a C\# DataSet object). If a non-XML file was requested, the binary information of the file is packaged within an XML file and returned. Translation services are also enabled whenever required from the technical services and are responsible for translating CAD files from their native source to a lightweight JT visualisation format.

System adapters: were developed to communicate the integration layer with data extracted from the domain layer. Due to the lack of integration capability and standardisation from the maintenance system involved in the automotive sector, system adapters are proposed to avoid the development of direct application program interface (API) calls that restrict the generality of the system. System adapters reduce the amount of effort required, when the system is implemented in a different domain. However, connecting to a new warranty system would still require some effort to customise the service depending on the API provided from the application. For enterprise systems such a PLM and enterprise resource planning (ERP) systems, there are adapters already available from each PLM and ERP vendor.

\subsubsection{Domain Layer}

The domain layer represents the business rules and the actual systems that will be used to extract data. It is critical that the domain layer be designed in isolation so it cannot be affected by the technology used. In order to achieve the level of integration required, system APIs are utilised to allow the communication among the layers and as a result the exchange of information.

Figure 6 shows the relationships among the different packages that are held within multiple silos. More importantly, it shows the types of data that the service needs to expose to the end users. These data sets are available from extraction from the service and can be served up to the end user whenever the right UI menu selection is applied. Any data manipulation such as the creation of Pareto charts will be handled either from the integration layer or the presentation layer once the data are cached within the aggregator.

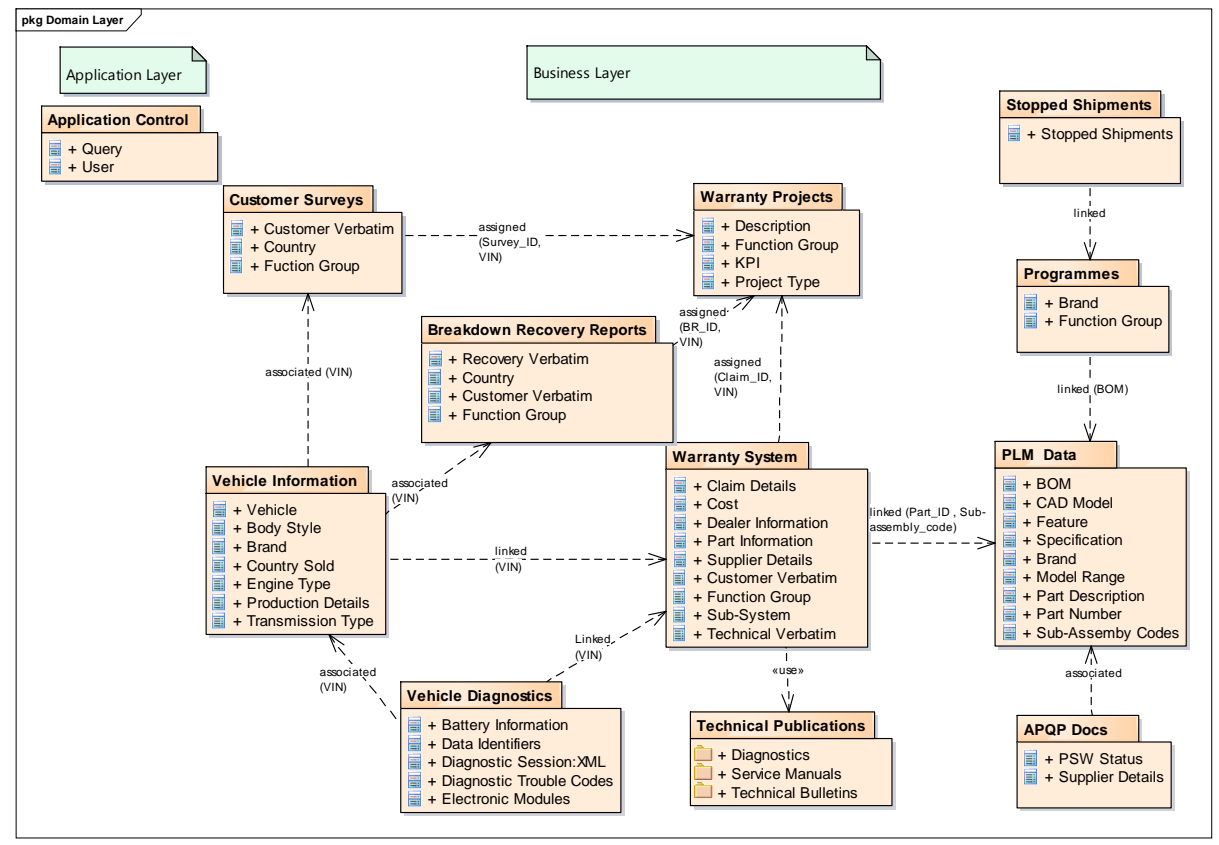

Figure 6 Domain Layer

\subsection{Dynamic view}

The main goal of the dynamic analysis is to model the dynamic behaviour of the system over time. Moreover, dynamic diagrams allow designers to verify the completeness and consistency of use cases [31]. Sequence and activity diagrams are widely used to model the dynamic view. 
In the two sequence diagrams presented in the "use case" view in Figs. 3 and 4, the proposed system appeared as a "black box". These two diagrams showed that a search term provides an input to the proposed solution which then utilises the search terms to extract the required data from all the systems integrated. Figure 7 shows the sequence of tasks and the messages exchanged within the system once a search term is received from the user and until the results are presented back to the user. Once the user logs in the system and defines the search criteria, the logic credentials and the search terms are passed to the workflow engine. The workflow engine will receive an input from the silo register to obtain the silo information. The workflow engine based on the search criteria and the login credentials will choose the most appropriate workflow. Therefore, the quality history for DFMEA and the root cause analysis follow different sequences of tasks within the system. The workflow engine obtains an input from the component mapping database with the correct primary keys which then uses to identify the relationships between the search criteria and the primary keys within each system. If additional information required, for example, for the vehicle diagnostics database, there might be a requirement to define a period of dates to reduce the number of results, and then the user is asked to fill additional search criteria. Otherwise, the workflow engine controls the technical services and executes the queries to the systems. The system initially requests the product structure from the PLM system which is returned in an XML format based on the part_ID and the sub_assembly_code defined within the search terms or extracted from the component mapping. Once the product structure is decomposed based on one parent and all children, the workflow engine requests through the technical services the extraction of all the data associated with the product structure and the other search terms (e.g. VIN). CAD models returned to the aggregator are translated into a JT format while all the other meta-data remain in an XML format. All the data are cached against a query number. The outcome of the system is a single evidence pack with all the relevant information which can be used as part of the root cause analysis.

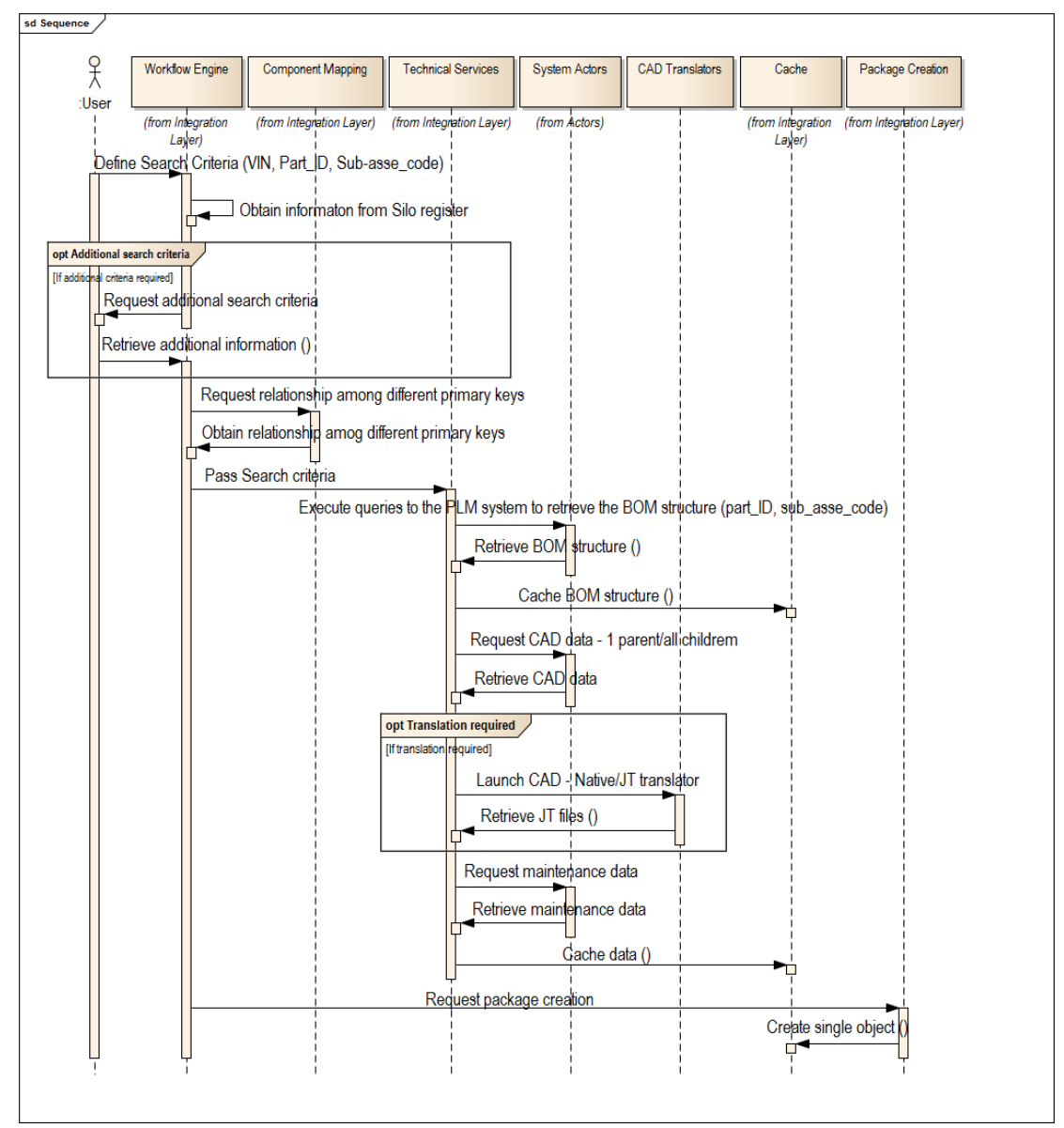

Figure 7 Dynamic view of the proposed system architecture

\subsection{Case Study}

This case study demonstrates how the system could be used by the OEM warranty engineers to assess a claim as described in the current state process, step 3. This case study was one of the three used to test and validate the system. Figures below are used for illustration purposes only and are not linked with real vehicle faults. What these scenarios aim to achieve is to demonstrate that by integrating product data from PLM systems with warranty claims, vehicle diagnostics and technical publications, engineers were able to improve the root cause analysis and close the information gaps. 
This case study is related to a fault in the mirror sub-assembly. In this instance, suppliers failed to replicate the symptom as the returned parts were fully functional when they were tested. OEM engineers investigated the fault further in order to identify the root cause of the issue. The proposed system was used to support the root cause analysis by providing all the related information and initiate the root cause analysis. The outcome of the system is an evidence pack that includes all the information related to this specific fault:

1. The OEM warranty engineer reloads the query that the supplier warranty engineer ran in order to load the data that suppliers used and assess the part. The system presents a generic visual representation of the vehicle and allows the user to colour code the vehicle representation based on different criteria such as areas where previous claims occurred for the searched VIN, cost of claims, etc. The system also presents a vehicle description of the search VIN (Fig. 8).

Vehicle's history such as claims, surveys and breakdown recovery reports associated with the searched VIN is also presented from the system. Table 1 shows the vehicle's history. The OEM warranty engineer reviews the vehicle's history to understand what the customer complaint is about, what the technician did to fix the issue and the conditions that the vehicle was operating (miles, country, etc.). In this example, the customer complained that the front side mirror is not folding with a key. The dealer checked the customer's concern and decided to remove and replace the front mirror side.

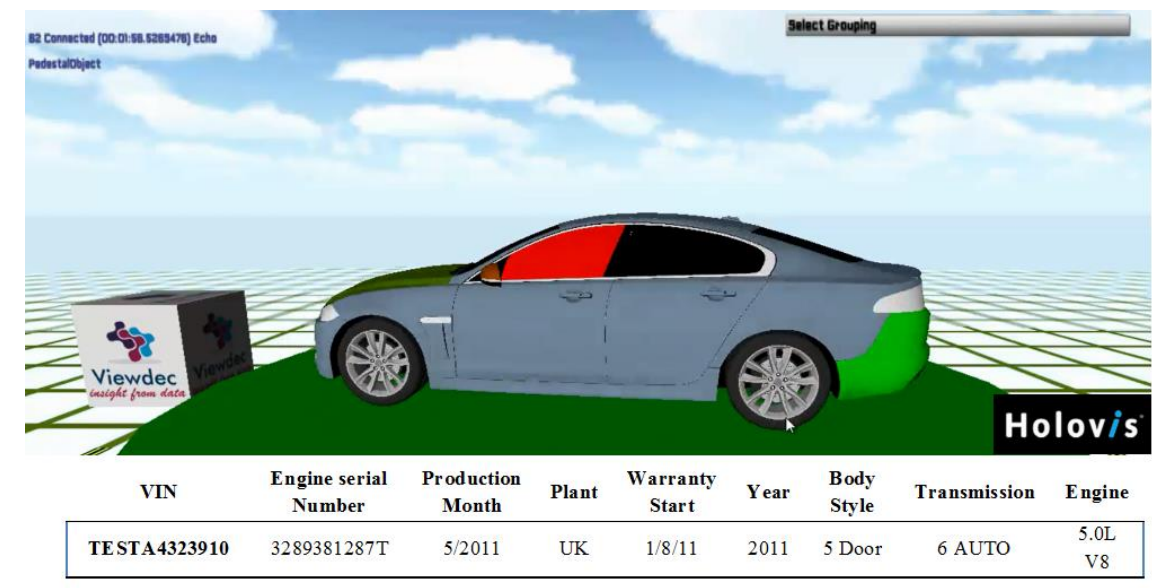

Figure 8 Vehicle description (Screen Shot Captured from Holovis UI)

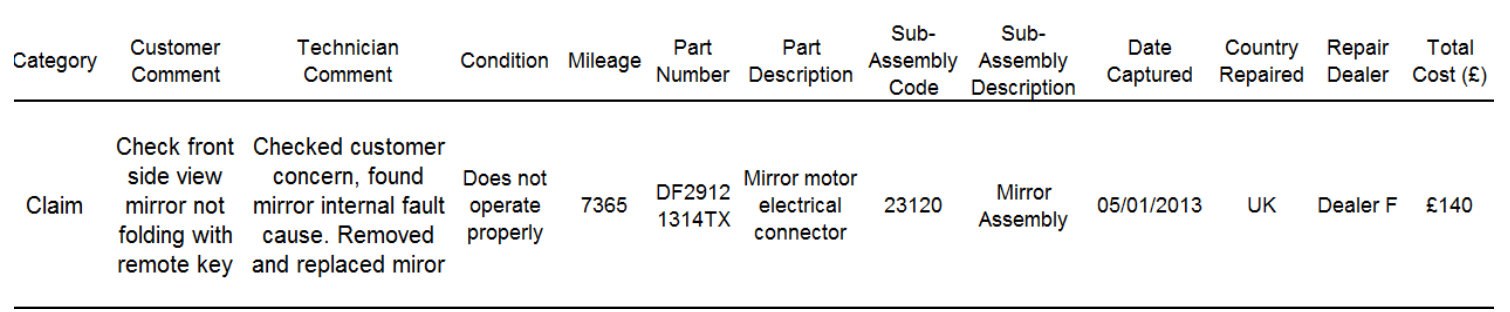

Table 1 Vehicle's History

2. The OEM warranty engineer selects to review the data associated with the claim. The system filters the vehicle history and presents only the information for the selected claim. A light-version CAD representation in a JT format is presented from the system.

3. As this is an issue related to electronic parts, the engineer selects from the menu option to review the DTC that the sensors captured. The system provides a description from the sensor that mirrors were unable to fold either due to "circuit short to battery" or due to an open electronic circuit.

4. The OEM warranty engineer reviews the CAD data and the documentation associated with the part to understand the interactions among the mirror and the other sub-systems used. The OEM warranty engineer reviews the tech documentation which suggests that all bulletins and special service messages are up to date. From the data analysed, there is a strong indication that this is probably related to a design issue. The diagnostics and the CAD assessment suggest that the plug within the mirror housing backs out slightly breaking the circuit. The engineer records the action and passes the evidence pack for further investigation to the engineering department. As an 
interim action, the engineer issues a special service message to the dealers to ensure that dealers will always check the plug prior to changing the part (Fig. 9).
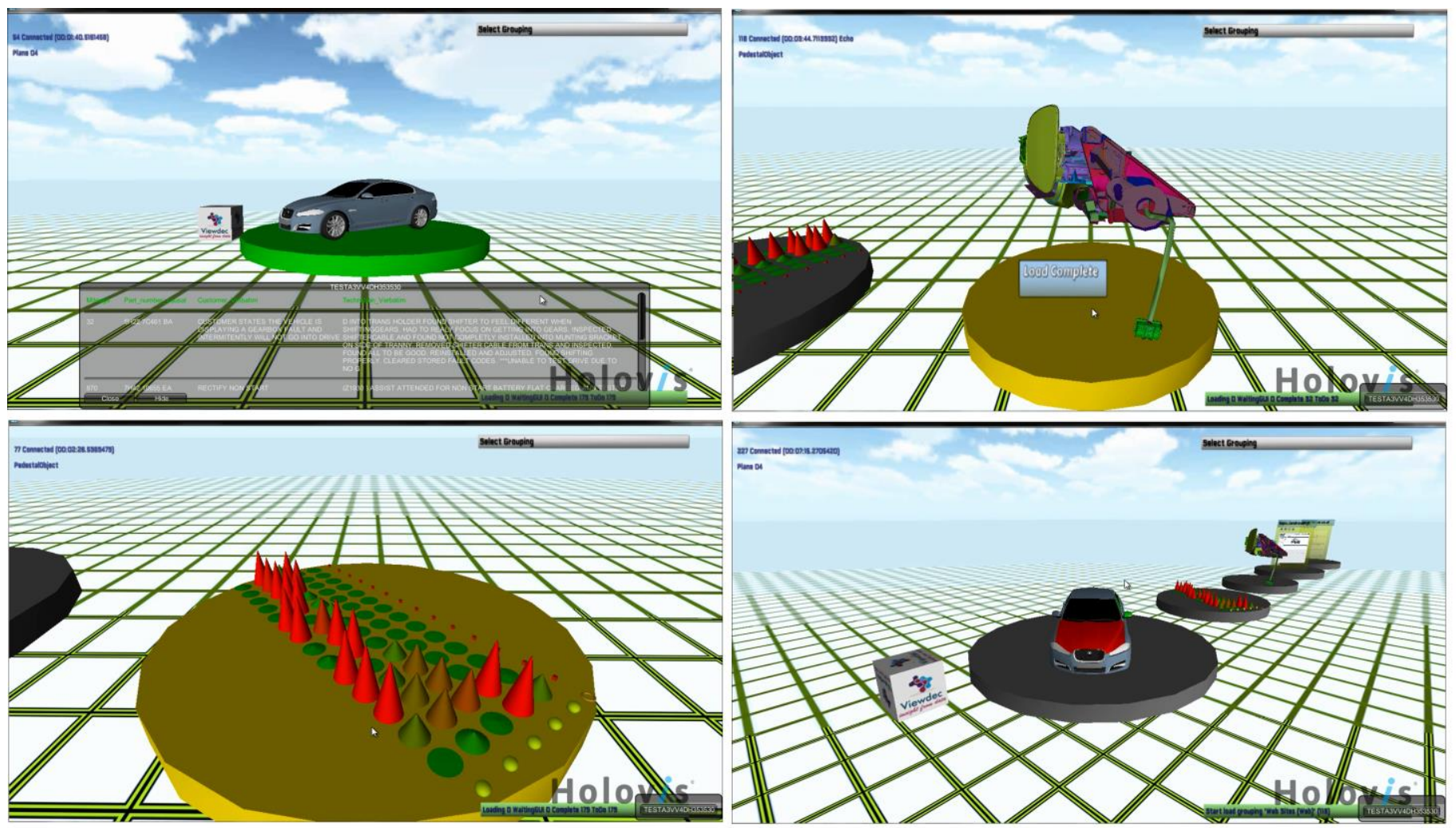

Figure 9 Different views of the Proof of Concept System (Screen Shots Captured from Holovis UI)

\section{Discussion and Future Directions}

This research demonstrated evidence of the benefits that can be achieved through the integration of service manuals, engineering documents, warranty claims and vehicle diagnostics which was an area that other researchers such as Chougule et al. [24] have recommended to extend their work into. The outcome of this research extends the findings of Lee et al. [7] further, not only by showing the potential of PLM systems within the maintenance procedures but also by demonstrating that through the integration of PLM systems with maintenance information, both PD engineers and warranty engineers could be benefited. All experts recognised the importance of integrating PLM systems with maintenance information and agreed that by reducing time and effort in analysing warranty claims and vehicle diagnostics, significant benefits can be achieved. By being able to analyse all the required information faster, engineers will be able to reduce the "time to detect" and as a result reduce the number of affected vehicles with the same fault. Another benefit that experts highlighted is the importance of having a standardised approach for root cause analysis across departments and suppliers within a single system. The proposed solution does not aim to replace the existing PLM and maintenance systems in companies but rather to provide a visualisation tool that enables OEMs and their supply chain to integrate and share accurate and complete information. This is one of the main contributions of this research as most of the systems proposed from researchers in the maintenance phase of the life cycle have a very internal and OEM focus. This research also demonstrated that information and knowledge coming back from maintenance experts and aggregated through the proposed system can also be used by PD engineers to prevent failures from recurring on new designed parts. Finally, this research proposes a solution that integrates disparate silos of data where data mining techniques could be applied later supporting the outcome of Buddhakulsomsiri et al.'s study.

From an infrastructure point of view, this research provides a web-based architecture that treats network as a virtual warehouse, removing the need to create centralised locations for managing maintenance data. The development of the proposed system architecture also highlights the importance of standards that can be used to improve system interoperability and reduce integration complexity. This is an area that the automotive sector can be benefited from the aerospace and the defence sectors were the development of standards is advanced.

Further research is required to develop algorithms that allow the system to combine vehicle diagnostics and warranty claims. The authors were unable to identify any existing work that proposes a model or algorithm that 
analyses warranty claims in relation to vehicle diagnostics. This is key for root cause analysis, considering that the amount of electronic parts in vehicles is continuously increasing. Further research is required to develop ways of structuring and analysing unstructured data from the web and social media. This area opens a new potential for the warranty root cause analysis as customer feedback from the web and social media can be received instantly providing engineers a vast amount of data such as pictures, videos and verbal descriptions of the fault.

\section{Conclusions}

The main outcome of this research is a novel system architecture for integrating PLM systems with cross supply chain maintenance information to support root cause analysis. The proposed solution was developed to support not only OEM and supplier warranty engineers to identify the root cause of an in-service product failure but also PD engineers who use the system to review the quality history of a part and ensure that previous failures will not reoccur to new product parts. The development of the system architecture was based on a structured methodology followed in order to identify the research gaps in relation to the current challenges that automotive engineers encounter. Data collection was achieved through a combination of semi-structured interviews with experts from the automotive sector, by studying the internal documentation and by testing the systems used.

The proposed architecture supports the review of all the required information associated with a claim in order to identify the root cause of an issue, close the information gaps and take precautionary actions to avoid fault reoccurrence on other vehicles. By maintaining the single source of truth philosophy, this approach ensures that the most up-to-date data are being used to aid the analysis and that by having links via web services to the original data, there is no danger of accessing stale copies held in centralised repositories. Moreover, it reduces the time spent searching for information by providing a centralised view of the data in a single environment. In addition, this system provides a standardised approach for root cause analysis across departments and suppliers. This is very important considering the number of people involved in this process. All the domain experts involved in supporting the development of the architecture recognised the importance of integrating PLM systems with warranty information and stated that being able to visualise a part in relation to its sub-assembly as well as having all the information required without going on each individual system can be very beneficial. This architecture was designed and tested with a sample data and is currently undergoing further testing before being implemented in a real production environment.

This research addressed the research gaps by demonstrating that (i) a system architecture can be developed to integrate PLM systems with maintenance information to allow the utilisation of knowledge and data across the product lifecycle, (ii) network can be treated as a virtual warehouse where maintenance data are integrated and shared within the supply chain, (iii) product data can be utilised in conjunction with maintenance information to support warranty and product development engineers, and (iv) disparate pieces of data can be integrated where later data mining techniques could potentially be applied.

Although the system is currently able to integrate all the required information into a single view, the analysis element is still complete on each data set individually. Further research is required to develop algorithms that allow the analysis of the different clusters of data together such as the vehicle diagnostics and the warranty claims. From a data integration point of view, further research is required to enable the standardisation of enterprise systems in order to improve collaboration among OEMs, suppliers and customers and as a result reduce the cost of implementing and maintaining multiple systems.

\section{Acknowledgements}

This research was conducted as part of 'VE-DRIVE' (Project no. 101060); a two year collaborative R\&D project which started in December 2011. This research was co-funded by Innovate UK, the UK's innovation agency and the project partners, Jaguar Land Rover, BAE Systems, Theorem Solutions and Holovis International with project management support by Axillium Research. The authors would like to thank all the project partners for their contribution to the project and especially the two software development companies, Theorem Solutions and Holovis International for developing the proof of concept system.

\section{Disclosure of potential conflicts of interest}

Funding: This research was funded as part of the VE-DRIVE project (Project no. 101060) and was co-funded by Innovate UK, Jaguar Land Rover and Axillium Research. 
Conflict of Interest: The authors have received research funding by Innovate UK, Jaguar Land Rover and Axillium

Research.

\section{References}

1. Buddhakulsomsiri J, Siradeghyan Y, Zakarian A, Li X (2006) Association rule-generation algorithm for mining automotive warranty data. Int J Prod Econ 44:2749-2770

2. Sairamesh J, Cohen M, Touma M, Padala D, Mohan R (2004)

Dealer collaboration: transforming the value chain through integration and relationships. Proc IEEE Int Conf E-Commer Technol CEC 2004:325

3. Mannar K, Ceglarek D, Niu F, Abifaraj B (2006) Fault region localization: product and process improvement based on field performance and manufacturing measurements. IEEE Trans Autom Sci Eng 3:423-439

4. Han T, Yang B (2006) Development of an e-maintenance system integrating advanced techniques. Comput Ind 57:569-580

5. Candell O, Karim R, Söderholm P (2009) eMaintenanceInformation logistics for maintenance support. Robot CIM-Int Manuf 25:937-944

6. CIMdata (2002) Empowering the future of business. CIM Data Report - Product Lifecycle Management

7. Lee SG, Ma Y, Thimm GL, Verstraeten J (2008) Product lifecycle management in aviation maintenance, repair and overhaul. Comput Ind 59:296303

8. Gulledge T, Hiroshige S, Iyer R (2010) Condition-based maintenance and the product improvement process. Comput Ind 61:813-832

9. Djamaludin D, Murthy D, Blischke W (1995) Bibliography on warranties. In: Blischke W, Murthy D (eds) Product Warranty Handbook. Marcel Dekker Inc, New York, pp 839-916

10. Wu S (2013) A review on coarse warranty data and analysis. Reliab Eng Syst Saf 114:1-11

11. Karim MR, Suzuki K (2005) Analysis of warranty claim data: a literature review. Qual Reliab Eng Int 22:667-686

12. Wu S (2012) Warranty data analysis: a review. Qual Reliab Eng Int 28:795-805

13. Li L (2000) Estimation of distribution functions using data from different environments. Lifetime Data Anal 6:271-279

14. Rai B, Singh N (2003) Hazard rate estimation from incomplete and unclean warranty data. Reliab Eng Syst Saf 81:79-92

15. Kim H, Rao BM (2000) Expected warranty cost of two-attribute free-replacement warranties based on a bivariate exponential distribution. Comput Ind Eng 38:425-434

16. Pal S, Murthy GSR (2003) An application of Gumbel's bivariate exponential distribution in estimation of warranty cost of motor cycles. Int J Qual Reliab Manag 20:488-502

17. Karim R, Yamamoto W, Suzuki K (2001) Statistical analysis of marginal count failure data. Lifetime Data Anal 7:173-186

18. Rai B, Singh N (2005) A modeling framework for assessing the impact of new time/mileage warranty limits on the number and cost of automotive warranty claims. Reliab Eng Syst Saf 88:157-169

19. Park M, Pham H (2012) Warranty cost analysis for k-out-of-n systems with 2-D warranty. IEEE Trans Syst Man Cybern A 42:947-957

20. Esterman M, Gerst P, Stiebitz PH, Ishil K (2005) A framework for warranty prediction during product development. ASME Des Eng 118:381

21. Sureka A, De S, Varma K (2008) Mining automotive warranty claims data for effective root cause analysis, Haritsa JR, Kotagiri R, Pudi V (ed). Database Systems for Advanced Application, New Delhi, pp 141-155

22. Choudhary AK, Harding JA, Tiwari MK (2009) Data mining in manufacturing: a review based on the kind of knowledge. J Intell Manuf 20:501521

23. Rajpathak DG (2013) An ontology based text mining system for knowledge discovery from the diagnosis data in the automotive domain. Comput Ind 64:565-580

24. Chougule R, Rajpathak D, Bandyopadhyay P (2011) An integrated framework for effective service and repair in the automotive domain: an application of association mining and case-based-reasoning. Comput Ind 62:742-754

25. Gusikhin O, Rychtyckyj N, Filev D (2007) Intelligent systems in the automotive industry: applications and trends. Knowl Inf Syst 12:147-168

26. Venkatasubramanian V (2005) Prognostic and diagnostic monitoring of complex systems for product lifecycle management: challenges and opportunities. Comput Chem Eng 29:1253-1263

27. Khare VR, Bandyopadhyay P, Waldo MB (2012) Automotive field failure analysis based on mileage - Feasibility \& benefits, PHM 2012 - 2012 I.E. Int. Conf. on Prognostics and Health Management: Enhancing Safety, Efficiency, Availability, and Effectiveness of Systems Through PHM Technology and Application, Conference Program, 18-21 June, pp. 1-8

28. Liu ZJ, Chen W, Huang H-Z, Yang B (2007) A diagnostics design decision model for products under warranty. Int J Prod Econ 109: 230-240

29. Saxena A, Wu B, Vachtsevanos G (2005) Integrated diagnosis and prognosis architecture for fleet vehicles using dynamic case-based reasoning. AUTOTESTCON (Proceedings): 96-102

30. Trowbridge D, Mancini D, Quick D, Hohpe G, Newkirk J, Lavigne D (2003) Enterprise solution patterns using microsoft. NET, patterns \& practices, 2nd ed, Microsoft

31. Cui Q, Liu C, Zha XF (2008) Intelligent drug delivery system using UML diagrams analysis. J Shanghai Jiaotong Uni (Science) 13: 312-317 
2015-09-21

\section{Improving root cause analysis through the integration of PLM systems with cross supply chain maintenance data}

\section{Madenas, $\mathrm{N}$}

Springer Verlag

Madenas, N., Tiwari, A., Turner, C.J. et al. Int J Adv Manuf Technol (2016) 84: 1679. doi:10.1007/s00170-015-7747-1 https://dspace.lib.cranfield.ac.uk/handle/1826/10768

Downloaded from Cranfield Library Services E-Repository 